\title{
On the General Consensus Protocol in Multiagent Networks with Double-Integrator Dynamics and Coupling Time Delay
}

\author{
Tao Dong ${ }^{1,2}$ and Xiaofeng Liao ${ }^{2}$ \\ ${ }^{1}$ College of Software and Engineering, Chongqing University of Posts and Telecommunications, \\ Chongqing 400065, China \\ ${ }^{2}$ College of Electronic and Information Engineering, Southwest University, Chongqing 400715, China \\ Correspondence should be addressed to Tao Dong; david_312@126.com and Xiaofeng Liao; 59508359@qq.com
}

Received 24 October 2012; Revised 6 January 2013; Accepted 25 January 2013

Academic Editor: Sheng-Jie Li

Copyright (C) 2013 T. Dong and X. Liao. This is an open access article distributed under the Creative Commons Attribution License, which permits unrestricted use, distribution, and reproduction in any medium, provided the original work is properly cited.

\begin{abstract}
This paper considers the problem of the convergence of the consensus algorithm for multiple agents in a directed network where each agent is governed by double-integrator dynamics and coupling time delay. The advantage of this protocol is that almost all the existing linear local interaction consensus protocols can be considered as special cases of the present paper. By combining algebraic graph theory and matrix theory and studying the distribution of the eigenvalues of the associated characteristic equation, some necessary and sufficient conditions are derived for reaching the second-order consensus. Finally, an illustrative example is also given to support the theoretical results.
\end{abstract}

\section{Introduction}

Recently, coordinated control of multiple agents has attracted a great deal of attention in many fields such as biology, physics, robotics, and control engineering $[1,2]$. Research on multiagent coordinated control problems not only helps in better understanding the general mechanisms and interconnection rules of natural collective phenomena, but also benefits many practical applications of networked cyberphysical systems, such as the coordination and control of distributed sensor networks [3], formation control in multirobots [4], unmanned autonomous vehicles (UAVs) formations $[5,6]$, flocking [7], complex networks $[8,9]$, and so on $[10,11]$. A critical problem in coordinated control of multiple agents is to find control laws such that all agents can reach an agreement regarding a certain quantity of interest that depends on the states of all agents. This problem is usually called the consensus problem [12]. In recent years, numerous studies have been conducted on this problem, some special second-order consensus protocols were presented, and some consensus conditions were obtained [13-18].

In the references on the consensus problem, multiagent systems with double-integrator dynamics have been paid great attentions because of their importance in practice.
In [13], the authors studied some necessary and sufficient conditions for second-order consensus multiagent system, and revealed that both the real and imaginary parts of the eigenvalues of the Laplacian matrix of the corresponding directed network play key roles in reaching consensus. In [14], Zhu et al. discussed the consensus problem of coupled second-order linear harmonic oscillators with local interaction, which means that the states of all agents converge to the same periodic function. In [15], Zhu discussed a more general linear form of consensus protocols for multiagent systems with double-integrator dynamics. In [16], Li et al. investigated the final consensus convergence state of multiagent dynamical systems by using a kind of generalized linear local interaction protocols without time delay. However, most of the protocols discussed in the existing literature have neither addressed the consensus of positive exponential dynamics, namely, each agent's state converges to a positive exponential function, nor considered the time delay in network. So, the observation provides us the motivation of this paper to investigate the consensus of second-order agents in directed networks double-integrators and coupling time delay.

In this paper, we consider the convergence of the secondorder consensus of multiagent systems composed of coupled double-integrators dynamics and coupling time delay. For 
this protocol, all the agents in the fixed directed network topology are governed by double-integrator dynamics. The advantage of this protocol is that almost all the existing linear local interaction consensus protocols can be considered as special cases of the present paper [13-16]. By combining algebraic graph theory and matrix theory and studying the distribution of the eigenvalues of the associated characteristic equation, we give explicitly the effect of delays for secondorder consensus. The contribution of this paper is to obtain a necessary and sufficient condition that the second-order consensus can be achieved in a delayed multiagent system with a directed spanning tree if and only if the time delay is less than a certain critical value.

The rest of this paper is outlined as follows. In Section 2, some preliminaries on the graph theory and the model formulation are given. The main results are established in Section 3. In Section 4, several numerical examples are simulated to verify the theoretical analysis. Conclusions are finally drawn in Section 5.

\section{Preliminaries}

In this subsection, some basic concepts and results about algebraic grapy theory are introduced. For more details about algebraic graph theory, please refer to [19]. Suppose that information exchange among agents in multiagent systems can be modeled by an interaction digraph. Let $g=(V, \varepsilon, A)$ denote a directed graph, where $V=\{1,2, \ldots, N\}$ denotes the node set, $\varepsilon \subseteq V \times V$ represents the edge set, and $A=\left(a_{i j}\right)_{N \times N}$ is the adjacency matrix. A directed edge $\varepsilon_{i j}$ in the network $g$ is denoted by the ordered pair of nodes $(i, j)$, where $i$ is the head and $j$ is the tail, which means that node $i$ can receive information from node $j$ [20]. The elements of the adjacency matrix $A$ are defined such that $a_{i j}=1 \Leftrightarrow \varepsilon_{i j} \in \varepsilon$, while $a_{i j}=0 \Leftrightarrow \varepsilon_{i j} \notin \varepsilon$. We always assume that there is no self-loop in network $g$; that is, $a_{i i}=0$ for all $i \in V$. Weighted adjacency matrix $A$ of a weighted directed graph can be defined such that $a_{i j}$ is a positive weight if $\varepsilon_{i j} \in \varepsilon$, while $a_{i j}=0$ if $\varepsilon_{i j} \notin \varepsilon$. The set of neighbors of node $i$ is denoted by $N_{i}=\{j \in V$ : $(i, j) \in \varepsilon\}$.

If there is a sequence of edges of the form $\left(i, j_{1}\right)$, $\left(j_{1}, j_{2}\right), \ldots,\left(j_{m}, j\right) \in \varepsilon$ composing a directed path beginning with $i$ and ending with $j$ in the directed graph $g$ with distinct nodes $j_{k}, k=1,2, \ldots, m$, then the node $j$ is said to be reachable from node $i$. A directed graph is strongly connected if for any distinct nodes $i$ and $j$, there exists a directed path from node $i$ to node $j$. A directed graph has a directed spanning tree if there exists at least one node called root which has a directed path to all the other nodes [21]. A directed graph is balanced if $\sum_{j=1}^{n} a_{i j}=\sum_{j=1}^{n} a_{j i}$ for all $i$. Let (generally nonsymmetrical) Laplacian matrix $L=$ $\left(l_{i j}\right)_{N \times N}$ associated with directed network $g$ be defined as $l_{i i}=\sum_{j=1, j \neq i}^{N} a_{i j}$ and $l_{i j}=-a_{i j}$, where $i \neq j$. Especially, for an undirected graph, $L$ is symmetric positive semidefinite. However, $L$ for a directed graph does not have this property.

2.1. Notations. Some mathematical notations are used for simplicity throughout this paper. $I_{n}\left(O_{n}\right)$ denotes the identity (zero) matrix with $n$ dimensions. Let $\mathbf{1}_{n}\left(\mathbf{0}_{n}\right)$ be a column vector with $n$ elements being $1(0) . \operatorname{Re}(\cdot)$ and $\operatorname{Im}(\cdot)$ represent the real part and imaginary part of a complex, respectively. $C^{n}$ is the $n$-dimensional complex vector space.

2.2. Consensus Protocols. Suppose that the $i$ th agent in the network $g$ is modeled by double-integrator dynamics and coupling time delay

$$
\dot{r}_{i}(t)=v_{i}(t), \quad \dot{v}_{i}(t)=u_{i}(t), \quad i=1,2, \ldots, N,
$$

where $r_{i}(t) \in R$ is the position state, $v_{i}(t) \in R$ is the velocity state, and $u_{i}(t) \in R$ is the control input, which is designed based on local information exchange. In this paper, the consensus protocol in a multiagents system is as follows:

$$
\begin{aligned}
u_{i}(t)= & -\alpha r_{i}(t)-\beta v_{i}(t)+\gamma \sum_{j \in N_{i}} a_{i j}\left(r_{j}(t-\tau)-r_{i}(t-\tau)\right) \\
& +\xi \sum_{j \in N_{i}} a_{i j}\left(v_{j}(t-\tau)-v_{i}(t-\tau)\right), \quad i=1,2, \ldots, N,
\end{aligned}
$$

where $\alpha>0(\beta>0)$ denotes the position (velocity) damping gain, $\gamma>0 \quad(\xi>0)$ represents the coupling strength of positions (velocities) between neighboring agents, and $\tau>$ 0 is the communication time-delay.

Equivalently, system (2) can be rewritten as follows:

$$
\begin{aligned}
u_{i}(t)= & -\alpha r_{i}(t)-\beta v_{i}(t)-\gamma \sum_{j \in N_{i}} L_{i j} r_{j}(t-\tau) \\
& -\xi \sum_{j \in N_{i}} L_{i j} v_{j}(t-\tau), \quad i=1,2, \ldots, N .
\end{aligned}
$$

Let $\mathbf{x}(t)=\left[r_{1}(t), r_{2}(t), \ldots, r_{N}(t)\right]^{T}$ and $\mathbf{v}(t)=$ $\left[v_{1}(t), v_{2}(t), \ldots, v_{N}(t)\right]^{T}$. Then, network (1) can be rewritten in a compact matrix form as

$$
\left[\begin{array}{c}
\dot{\mathbf{x}}(t) \\
\dot{\mathbf{v}}(t)
\end{array}\right]=Q\left[\begin{array}{c}
\mathbf{x}(t) \\
\mathbf{v}(t)
\end{array}\right]
$$

where $Q=\left[\begin{array}{cc}O_{N} & I_{N} \\ -\alpha I_{N}-\gamma L e^{-\lambda \tau} & -\beta I_{N}-\xi L e^{-\lambda \tau}\end{array}\right]$, and $L \in R^{N \times N}$ is the (nonsymmetric) Laplacian matrix associated with directed graph $g$.

Definition 1. Second-order consensus in the multiagent system (1) under control input (2) is said to be achieved if for any initial conditions $r_{i}(0), v_{i}(0), \lim _{t \rightarrow \infty}\left|r_{i}(t)-r_{j}(t)\right|=0$ and $\lim _{t \rightarrow \infty}\left|v_{i}(t)-v_{j}(t)\right|=0, \forall i, j=1,2, \ldots, N$.

\section{Main Results}

In delayed systems, the time delays can be regarded as the bifurcation parameters. In $[17,18]$, it was found that Hopf bifurcation occurs when time delays pass through some critical values where the conditions for local asymptotical stability of the equilibrium are not satisfied. Similarly, in this section, we aim to find the maximum time delay with which the consensus can be achieved in the multiagent system (1). Before reaching our main results, we first give the following lemmas. 
Lemma 2 (see [5]). Let $L$ be the (nonsymmetric) Laplacian matrix associated with directed network $g$. Then, L has a simple zero eigenvalue and its other eigenvalues have positive real parts if and only if $g$ has a directed spanning tree.

The characteristic equation for system (4) is

$$
\begin{aligned}
\operatorname{det} & {\left[\begin{array}{cc}
\lambda I_{N} & -I_{N} \\
\alpha I_{N}+\gamma L e^{-\lambda \tau} & \lambda I_{N}+\beta I_{N}+\xi L e^{-\lambda \tau}
\end{array}\right] } \\
= & \operatorname{det}\left(\lambda I_{N}\left(\lambda I_{N}+\beta I_{N}+\xi L e^{-\lambda \tau}\right)+\alpha I_{N}+\gamma L e^{-\lambda \tau}\right) \\
= & \operatorname{det}\left(\lambda^{2} I_{N}+\beta \lambda I_{N}+\alpha I_{N}+e^{-\lambda \tau}\left(\lambda I_{N} \xi L+\gamma L\right)\right) \\
= & \prod_{i=1}^{N}\left[\lambda^{2}+\beta \lambda+\alpha-\left(\lambda \xi \mu_{i}+\gamma \mu_{i}\right) e^{-\lambda \tau}\right] \\
= & 0 .
\end{aligned}
$$

Let $g_{i}(\lambda)=\lambda^{2}+\beta \lambda+\alpha-\left(\lambda \xi \mu_{i}+\gamma \mu_{i}\right) e^{-\lambda \tau}$ and $g(\lambda)=\prod_{i=1}^{N} g_{i}(\lambda)$. From (5), it is easy to see that $L$ has a zero eigenvalue of algebraic multiplicity $m$ if and only if $g(\lambda)=0$ has a zero eigenvalue of algebraic multiplicity $2 \mathrm{~m}$.

In the following, one considers the case that $g_{i}(\lambda)$ has a pair of purely imaginary roots $\pm i \omega_{i}$. If $i \omega_{i}\left(\omega_{i}>0\right)$ is a root of $g_{i}(\lambda)=0$, substituting it into $g_{i}(\lambda)$, one can obtain

$$
-\omega_{i}^{2}+\beta i \omega_{i}+\alpha-\left(i \omega_{i} \xi \mu_{i}+\gamma \mu_{i}\right) e^{-i \omega \tau}=0
$$

Separating the real and imaginary parts of (6) leads to

$$
\begin{aligned}
& {\left[R\left(\mu_{i}\right) \gamma-l\left(\mu_{i}\right) \omega_{i} \xi\right] \cos \omega_{i} \tau+\left[R\left(\mu_{i}\right) \omega_{i} \xi+l\left(\mu_{i}\right) \gamma\right] \sin \omega_{i} \tau} \\
& \quad=-\omega_{i}^{2}+\alpha, \\
& {\left[R\left(\mu_{i}\right) \omega_{i} \xi+l\left(\mu_{i}\right) \gamma\right] \cos \omega_{i} \tau-\left[R\left(\mu_{i}\right) \gamma-l\left(\mu_{i}\right) \omega_{i} \xi\right] \sin \omega_{i} \tau} \\
& \quad=\beta \omega_{i} .
\end{aligned}
$$

By squaring and adding the previous equations, it follows that

$$
\omega_{i}^{4}-\left(\left\|\mu_{i}\right\|^{2} \xi^{2}+2 \alpha-\beta^{2}\right) \omega_{i}^{2}-\left\|\mu_{i}\right\|^{2} \gamma^{2}+\alpha^{2}=0 .
$$

By solving (8), one can obtain

$$
=\frac{\left\|\mu_{i}\right\|^{2} \xi^{2}+2 \alpha-\beta^{2} \pm \sqrt{\left(\left\|\mu_{i}\right\|^{2} \xi^{2}+2 \alpha-\beta^{2}\right)^{2}+4\left(\left\|\mu_{i}\right\|^{2} r^{2}-\alpha^{2}\right)}}{2} .
$$

Lemma 3. (1) If $\left\|\mu_{i}\right\|^{2} \xi^{2}+2 \alpha<\beta^{2}$ and $\left\|\mu_{i}\right\|^{2} \gamma^{2}<\alpha^{2}$, then (8) has no positive root.

(2) If $\left\|\mu_{i}\right\|^{2} \xi^{2}+2 \alpha>\beta^{2}$ and $\left\|\mu_{i}\right\|^{2} \gamma^{2}>\alpha^{2}$, then (8) has only one positive root.

(3) If $\left\|\mu_{i}\right\|^{2} \xi^{2}+2 \alpha>\beta^{2}$ and $\left\|\mu_{i}\right\|^{2} \gamma^{2}<\alpha^{2}$, then (8) has two positive roots.
Suppose that (8) has positive roots; without loss of generality, one assumes that it has two positive roots defined by $\omega_{i k}$, $(k=1,2)$. By (7), one has

$$
\begin{aligned}
& \cos \left(\omega_{i k} \tau\right) \\
& =\frac{\left(-\omega_{i k}^{2}+\alpha\right)\left[R\left(\mu_{i}\right) \gamma-l\left(\mu_{i}\right) \omega_{i k} \xi\right]+\beta \omega_{i k}\left[R\left(\mu_{i}\right) \omega_{i k} \xi+l\left(\mu_{i}\right) \gamma\right]}{\left(\omega_{i k}^{2}-\alpha\right)^{2}+\beta^{2} \omega_{i k}^{2}}
\end{aligned}
$$

Thus, denoting

$$
\begin{aligned}
& \tau_{i k}^{j} \\
& =\frac{1}{\omega_{i k}} \arccos \frac{\left(-\omega_{i k}^{2}+\alpha\right)\left[R\left(\mu_{i}\right) \gamma-l\left(\mu_{i}\right) \omega_{i k} \xi\right]+\beta \omega_{i k}\left[R\left(\mu_{i}\right) \omega_{i k} \xi+l\left(\mu_{i}\right) \gamma\right]}{\left(\omega_{i k}^{2}-\alpha\right)^{2}+\beta^{2} \omega_{i k}^{2}} \\
& \quad+\frac{2 j \pi}{\omega_{i k}},
\end{aligned}
$$

where $j=0,1, \ldots$, then $\pm i \omega_{i}$ is a pair of purely imaginary roots of $g_{i}(\lambda)=0$ with $\tau_{i k}^{j}$

$$
\text { Define } \tau_{i}^{0}=\min _{k=1,2}\left\{\tau_{i k}\right\} \text { and } \tau^{0}=\min _{i=2, \ldots, N}\left\{\tau_{i}^{0}\right\} \text {. }
$$

Lemma 4 (see [15]). Consider the exponential polynomial

$$
\begin{aligned}
P\left(\lambda, e^{-\lambda \tau}, \ldots, e^{-\lambda \tau_{m}}\right) \\
=\lambda^{n}+p_{1}^{(0)} \lambda^{n-1}+\cdots+p_{n-1}^{(0)} \lambda+p_{n}^{(0)} \\
\quad+\left[p_{1}^{(1)} \lambda^{n-1}+\cdots+p_{n-1}^{(1)} \lambda+p_{n}^{(1)}\right] e^{-\lambda \tau} \\
\quad+\cdots+\left[p_{1}^{(m)} \lambda^{n-1}+\cdots+p_{n-1}^{(m)} \lambda+p_{n}^{(m)}\right] e^{-\lambda \tau_{m}},
\end{aligned}
$$

where $\tau_{i} \geq 0(i=1,2, \ldots, m)$ and $p_{j}^{(i)}(j=1,2, \ldots, m)$ are constants. As $\left(\tau_{1}, \tau_{2}, \ldots, \tau_{m}\right)$ vary, the sum of the order of the zeros of $P\left(\lambda, e^{-\lambda \tau}, \ldots, e^{-\lambda \tau_{m}}\right)$ on the open right half plane can change only if a zero appears on or crosses the imaginary axis. Let

$$
\begin{aligned}
\delta=\max _{2 \leq i \leq N}\{ & -2 \alpha \beta^{2}+4 \operatorname{Re}\left(\mu_{i}\right) \alpha \beta \xi-2 \operatorname{Re}^{2}\left(\mu_{i}\right) \alpha \xi^{2} \\
& -2 \operatorname{Re}\left(\mu_{i}\right) \beta^{3} \xi+2 \operatorname{Re}\left(\mu_{i}\right) \beta^{2} \gamma \\
& +\operatorname{Re}^{2}\left(\mu_{i}\right) \beta^{2} \xi^{2}-2 \operatorname{Re}^{3}\left(\mu_{i}\right) \beta \xi^{3} \\
& -2\left[2 \operatorname{Re}^{2}\left(\mu_{i}\right)+\operatorname{Im}^{2}\left(\mu_{i}\right)\right] \beta \gamma \xi \\
& +2 \operatorname{Re}\left(\mu_{i}\right)\left[\operatorname{Re}^{2}\left(\mu_{i}\right)+\operatorname{Im}^{2}\left(\mu_{i}\right)\right] \xi^{2} \gamma \\
& \left.+2 \operatorname{Im}^{2}\left(\mu_{i}\right) \gamma^{2}\right\} .
\end{aligned}
$$

Lemma 5. Suppose that the network contains a directed spanning tree, and all the roots of (5) have negative real parts if and only if the following conditions are satisfied: $\delta<0$ and $\tau \in\left[0, \tau_{0}\right)$.

Proof. For $\tau=0$, (5) can be rewritten in the following form:

$$
\prod_{i=1}^{N}\left[\lambda^{2}+\left(\beta-\xi \mu_{i}\right) \lambda+\alpha-\gamma \mu_{i}\right]=0 .
$$


Therefore, the roots of (14) satisfy

$$
\lambda^{2}+\left(\beta-\xi \mu_{i}\right) \lambda+\alpha-\gamma \mu_{i}=0 .
$$

We know that each eigenvalue of $-L, \mu_{i}$, corresponds to two eigenvalues of $Q$, denoted by $\lambda_{i \pm}$. Their relationships can be described by

$$
\begin{array}{r}
\lambda_{i \pm}=\frac{-\beta+\xi \mu_{i} \pm \sqrt{\left(\beta-\xi \mu_{i}\right)^{2}-4\left(\alpha-\gamma \mu_{i}\right)}}{2}, \\
i=1,2, \ldots, N .
\end{array}
$$

From Lemma 2, one knows that $-L$ has a simple zero eigenvalue, and all the other eigenvalues have negative parts if and only if the directed network $g$ has a directed spanning tree. That is to say, $\operatorname{Re}\left(\lambda_{i \pm}\right)<0, i=2, \ldots, N$. For convenience, let $\sqrt{\left(\beta-\xi \mu_{i}\right)^{2}-4\left(\alpha-\gamma \mu_{i}\right)}=c+\mathbf{i} d$, where $c, d$ are real numbers, and $\mathbf{i}=\sqrt{-1}$. It follows from (16) that $\operatorname{Re}\left(\lambda_{i+}\right)<0$ implies $\left(-\beta+\xi \operatorname{Re}\left(\mu_{i}\right) \pm c\right) / 2<0, i=2, \ldots, N$. This is equivalent to the following two inequalities $\xi \operatorname{Re}\left(\mu_{i}\right)-\beta<$ $c<-\xi \operatorname{Re}\left(\mu_{i}\right)+\beta$ and $\operatorname{Re}\left(\mu_{i}\right)<0$ which hold simultaneously. Therefore,

$$
c^{2}<\xi^{2} \operatorname{Re}^{2}\left(\mu_{i}\right)+\beta^{2}-2 \xi \beta \operatorname{Re}\left(\mu_{i}\right), \quad i=2,3, \ldots, N .
$$

Also note that $\left(\beta-\xi \mu_{i}\right)^{2}-4\left(\alpha-\gamma \mu_{i}\right)=c^{2}-d^{2}+\mathbf{i} 2 c d$. By some calculations and separating the real and imaginary parts, one obtains

$$
\begin{gathered}
\beta^{2}+\xi^{2} \operatorname{Re}^{2}\left(\mu_{i}\right)-\xi^{2} \operatorname{Im}^{2}\left(\mu_{i}\right)-2 \beta \xi \operatorname{Re}\left(\mu_{i}\right) \\
-4 \alpha+4 \gamma \operatorname{Re}\left(\mu_{i}\right)=c^{2}-d^{2}, \\
\xi^{2} \operatorname{Re}\left(\mu_{i}\right) \operatorname{Im}\left(\mu_{i}\right)-\beta \xi \operatorname{Im}\left(\mu_{i}\right)+2 \gamma \operatorname{Im}\left(\mu_{i}\right)=c d .
\end{gathered}
$$

It follows from (18) that

$$
c^{4}-B c^{2}-A=0,
$$

where

$$
\begin{aligned}
B= & \beta^{2}+\xi^{2} \operatorname{Re}^{2}\left(\mu_{i}\right)-\xi^{2} \operatorname{Im}^{2}\left(\mu_{i}\right) \\
& -2 \beta \xi \operatorname{Re}\left(\mu_{i}\right)-4 \alpha+4 \gamma \operatorname{Re}\left(\mu_{i}\right), \\
A= & {\left[\xi^{2} \operatorname{Re}\left(\mu_{i}\right) \operatorname{Im}\left(\mu_{i}\right)-\beta \xi \operatorname{Im}\left(\mu_{i}\right)+2 \gamma \operatorname{Im}\left(\mu_{i}\right)\right]^{2} . }
\end{aligned}
$$

Solving (19) and combining (17) yield

$$
\begin{array}{r}
c^{2}=\frac{B \pm \sqrt{B^{2}+4 A}}{2}<\xi^{2} \operatorname{Re}^{2}\left(\mu_{i}\right)+\beta^{2}-2 \xi \beta \operatorname{Re}\left(\mu_{i}\right) \\
i=2,3, \ldots, N .
\end{array}
$$

After some simplifications, we can derive that the inequality $\delta<0$ in Lemma 5 holds. Using Lemma 4 and similar to the approach in $[22,23]$, we complete the proof.

Summarizing the previous discussions, we have the following theorem.

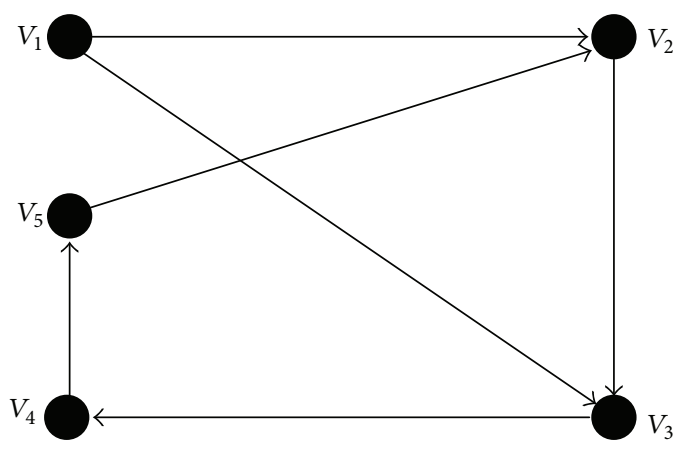

Figure 1: The directed interaction topology of five agents.

Theorem 6. Suppose that the network contains a directed spanning tree; we have the following results.

(1) If $\max \left\{\left\|\mu_{i}\right\|^{2} \xi^{2}+2 \alpha\right\}<\beta^{2}, \max \left\{\left\|\mu_{i}\right\|^{2} r^{2}\right\}<\alpha^{2}$, and $\delta<$ 0 , the second-order consensus in system (1) is achieved.

(2) If $\min \left\{\left\|\mu_{i}\right\|^{2} \xi^{2}+2 \alpha\right\}>\beta^{2}, \delta<0$ and $\tau \in\left[0, \tau_{0}\right)$, the second-order consensus in system (1) is achieved.

Corollary 7. For $\alpha=\beta=0, \gamma, \xi>0$, and $\tau>0$, second-order consensus in multiagent system (1) can be achieved if and only if the network contains a directed spanning tree and

$$
\begin{array}{r}
\frac{\xi^{2}}{\gamma}>\max _{2 \leq i \leq N}\left\{\frac{\operatorname{Im}^{2}\left(\mu_{i}\right)}{-\operatorname{Re}\left(\mu_{i}\right)\left[\operatorname{Re}^{2}\left(\mu_{i}\right)+\operatorname{Im}^{2}\left(\mu_{i}\right)\right]}\right\}, \\
\tau<\tau_{0}=\min _{2 \leq i \leq N}\left\{\frac{\theta_{i 1}}{\omega_{i 1}}\right\},
\end{array}
$$

where $\mu_{i}$ are nonzero eigenvalues of matrix $-L, i=2,3, \ldots, N$. In [13], Yu et al. studied the second-order consensus under the linear protocol:

$$
\begin{array}{r}
u_{i}(t)=\gamma \sum_{j \in N_{i}} a_{i j}\left(r_{j}(t)-r_{i}(t)\right)+\xi \sum_{j \in N_{i}} a_{i j}\left(v_{j}(t)-v_{i}(t)\right), \\
i=1,2, \ldots, N,
\end{array}
$$

and obtained the similar conclusions as Corollary 7. Therefore, Theorem 6 can be viewed as the extension of the results in [13].

Corollary 8. For $\tau=0$, second-order consensus in multiagent system (1) can be achieved if and only if the network contains a directed spanning tree and $\delta<0$. In [15], Zhu investigated the second-order consensus under the linear protocol:

$$
\begin{aligned}
u_{i}(t)= & -\alpha r_{i}(t)-\beta v_{i}(t)+\gamma \sum_{j \in N_{i}} a_{i j}\left(r_{j}(t)-r_{i}(t)\right) \\
& +\xi \sum_{j \in N_{i}} a_{i j}\left(v_{j}(t)-v_{i}(t)\right), \quad i=1,2, \ldots, N,
\end{aligned}
$$




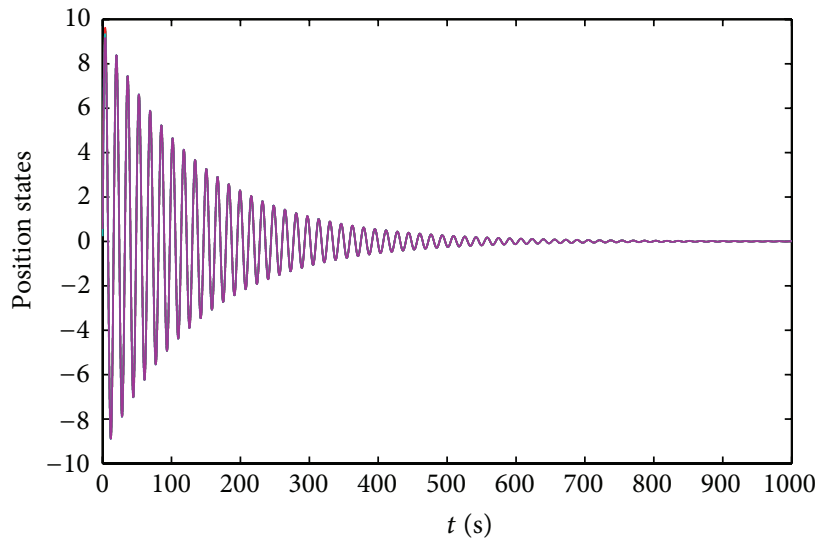

(a)

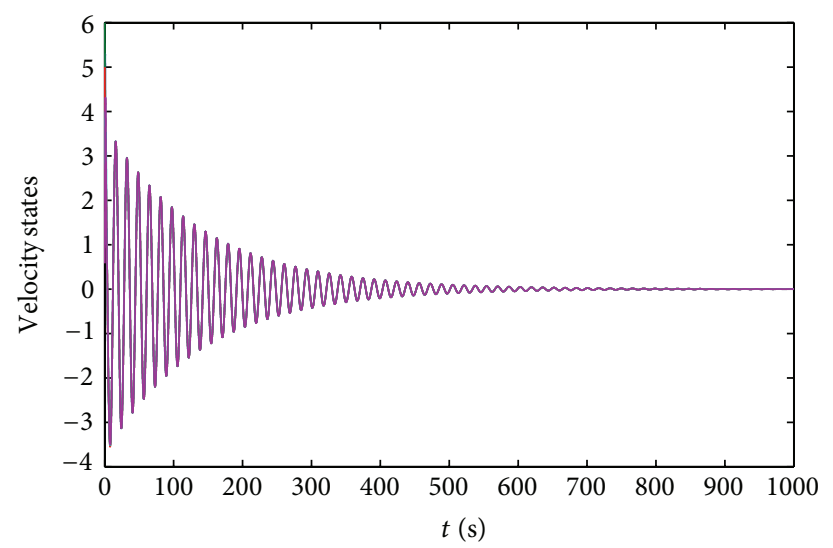

(b)

FIGURE 2: Velocity and position states of five agents in a network under linear consensus protocols where $\tau=0.39<\tau_{0}$.

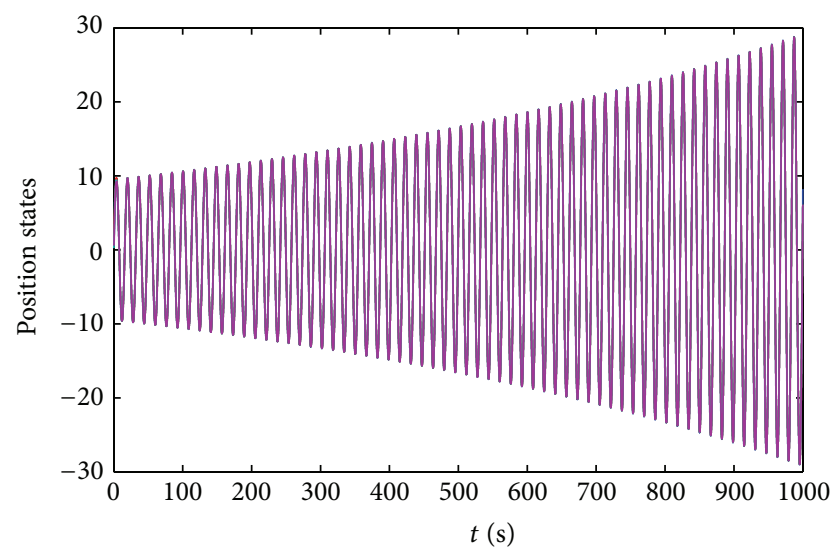

(a)

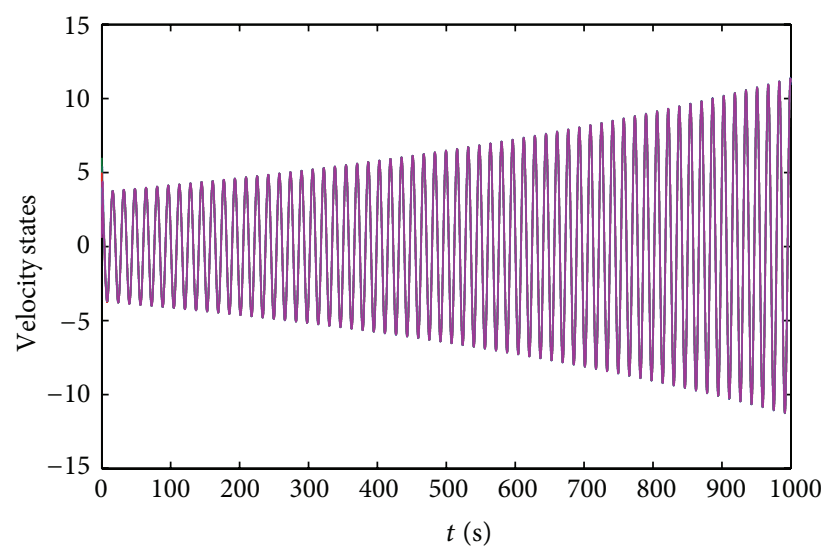

(b)

FIGURE 3: Velocity and position states of five agents in a network under periodic consensus protocols where $\tau=0.394>\tau_{0}$.

and obtained the similar conclusions as Corollary 8. Therefore, Theorem 6 can be viewed as the extension of the results in [15].

\section{Simulation Examples}

In this section, some numerical results of simulating system (1) are given to verify the theorems obtained earlier. Consider the network (1) with the topology shown in Figure 1. In Figure 1, the adjacency matrix $A$ and the Laplacian matrix $L$ of directed network $g$ are given in (25). Moreover, it is easily to see that there exists a directed spanning tree Figure 1. With simple calculations, we obtain the eigenvalues of $-L$, $\mu_{1}=0, \mu_{2}=-1+\mathbf{i}, \mu_{3}=-1-\mathbf{i}, \mu_{4}=-2$, and $\mu_{5}=-2$. Consider

$$
A=\left[\begin{array}{lllll}
0 & 1 & 1 & 0 & 0 \\
0 & 0 & 1 & 0 & 0 \\
0 & 0 & 0 & 1 & 0 \\
0 & 0 & 0 & 0 & 1 \\
0 & 1 & 0 & 0 & 0
\end{array}\right],
$$

$$
L=\left[\begin{array}{ccccc}
2 & -1 & -1 & 0 & 0 \\
0 & 1 & -1 & 0 & 0 \\
0 & 0 & 1 & -1 & 0 \\
0 & 0 & 0 & 1 & -1 \\
0 & -1 & 0 & 0 & 1
\end{array}\right]
$$

Let us set the parameters of the system (1) as follows: $\alpha=$ $0.1, \beta=0.2, \gamma=0.5$, and $\xi=1$. According to (11) and Lemma 5, we obtain $\tau_{0}=0.3939$ and $\delta=-0.3962<0$.

Consider $\tau=0.39<\tau_{0}$; from Theorem 6 , we know that the second-order consensus can be reached. The position and velocity states of all the agents are shown in Figure 2. It is easy to see that the consensus of all agents is achieved.

Consider $\tau=0.394>\tau_{0}$; from Theorem 6, we know that the second-order consensus cannot be reached. The position and velocity states of all the agents are shown in Figure 3. It is easy to see that the consensus of all agents is not achieved. 


\section{Conclusions}

In this paper, the convergence of the second-order consensus of multiagent systems composed of coupled doubleintegrators dynamics and coupling time delay has been studied. The advantage of the considered protocol is that it can be treated as the extensions of most linear local interaction protocols in the existing literatures. By combining algebraic graph theory and matrix theory and studying the distribution of the eigenvalues of the associated characteristic equation, some necessary and sufficient conditions are derived for reaching the second-order consensus. Several simulation results further validate the effectiveness of theoretical analysis.

\section{Acknowledgments}

This work was supported in part by Project no. CDJXS12180002 supported by the Fundamental Research Funds for the Central Universities, in part by the National Natural Science Foundation of China under Grant 60973114 and Grant 61170249, in part by the Research Fund of Preferential Development Domain for the Doctoral Program of Ministry of Education of China under Grant 201101911130005, in part by the State Key Laboratory of Power Transmission Equipment \& System Security and New Technology, Chongqing University, under Grant 2007DA10512711206, and in part by the Program for Changjiang Scholars.

\section{References}

[1] J. Hu and Y. Hong, "Leader-following coordination of multiagent systems with coupling time delays," Physica A, vol. 374, no. 2, pp. 853-863, 2007.

[2] W. Ren, R. W. Beard, and E. M. Atkins, "Information consensus in multivehicle cooperative control," IEEE Control Systems Magazine, vol. 27, no. 2, pp. 71-82, 2007.

[3] W. Yu, G. Chen, Z. Wang, and W. Yang, "Distributed consensus filtering in sensor networks," IEEE Transactions on Systems, Man, and Cybernetics B, vol. 39, no. 6, pp. 1568-1577, 2009.

[4] A. K. Das, R. Fierro, V. Kumar, J. P. Ostrowski, J. Spletzer, and C. J. Taylor, "A vision-based formation control framework," IEEE Transactions on Robotics and Automation, vol. 18, no. 5, pp. 813825, 2002.

[5] B. D. O. Anderson, B. Fidan, C. Yu, and D. van der Walle, "UAV formation control: theory and application," in Recent Advances in Learning and Control, vol. 371 of Lecture Notes in Control and Information Sciences, pp. 15-33, Springer, London, UK, 2008.

[6] B. Vanek, T. Péni, J. Bokor, and G. Balas, "Practical approach to real-time trajectory tracking of UAV formations," in Proceedings of the American Control Conference (ACC '05), pp. 122-127, usa, June 2005.

[7] R. Olfati-Saber, "Flocking for multi-agent dynamic systems: algorithms and theory," IEEE Transactions on Automatic Control, vol. 51, no. 3, pp. 401-420, 2006.

[8] J. Lu, D. W. C. Ho, and L. Wu, "Exponential stabilization of switched stochastic dynamical networks," Nonlinearity, vol. 22, no. 4, pp. 889-911, 2009.

[9] J. Lu, D. W. C. Ho, and Z. Wang, "Pinning stabilization of linearly coupled stochastic neural networks via minimum number of controllers," IEEE Transactions on Neural Networks, vol. 20, no. 10, pp. 1617-1629, 2009.

[10] W. Yu, G. Chen, and M. Cao, "Consensus in directed networks of agents with nonlinear dynamics," IEEE Transactions on Automatic Control, vol. 56, no. 6, pp. 1436-1441, 2011.

[11] P. Lin and Y. Jia, "Consensus of second-order discrete-time multi-agent systems with nonuniform time-delays and dynamically changing topologies," Automatica, vol. 45, no. 9, pp. 21542158, 2009.

[12] W. Ren and R. W. Beard, "Consensus seeking in multiagent systems under dynamically changing interaction topologies," IEEE Transactions on Automatic Control, vol. 50, no. 5, pp. 655-661, 2005.

[13] W. Yu, G. Chen, and M. Cao, "Some necessary and sufficient conditions for second-order consensus in multi-agent dynamical systems," Automatica, vol. 46, no. 6, pp. 1089-1095, 2010.

[14] J. Zhu, Y.-P. Tian, and J. Kuang, "On the general consensus protocol of multi-agent systems with double-integrator dynamics," Linear Algebra and its Applications, vol. 431, no. 5-7, pp. 701-715, 2009.

[15] J. Zhu, "On consensus speed of multi-agent systems with double-integrator dynamics," Linear Algebra and its Applications, vol. 434, no. 1, pp. 294-306, 2011.

[16] H. Li, X. Liao, T. Dong, and L. Xiao, "Second-order consensus seeking in directed networks of multi-agent dynamical systems via generalized linear local interaction protocols," Nonlinear Dynamics, vol. 70, pp. 2213-2226, 2012.

[17] T. Dong, X. Liao, and T. Huang, "Dynamics of a congestion control model in a wireless access network," Nonlinear Analysis. Real World Applications, vol. 14, no. 1, pp. 671-683, 2013.

[18] T. Dong, X. Liao, and H. Li, "Stability and Hopf bifurcation in a computer virus model with multistate antivirus," Abstract and Applied Analysis, Article ID 841987, 16 pages, 2012.

[19] C. Godsil and G. Royle, Algebraic Graph Theory, vol. 207 of Graduate Texts in Mathematics, Springer, New York, NY, USA, 2001.

[20] W. Zhu and D. Cheng, "Leader-following consensus of secondorder agents with multiple time-varying delays," Automatica, vol. 46, no. 12, pp. 1994-1999, 2010.

[21] Q. Song, J. Cao, and W. Yu, "Second-order leader-following consensus of nonlinear multi-agent systems via pinning control," Systems \& Control Letters, vol. 59, no. 9, pp. 553-562, 2010.

[22] T. Dong, X. Liao, T. Huang, and H. Li, "Hopf-pitchfork bifurcation in an inertial two-neuron system with time delay," Neurocomputing, vol. 97, pp. 223-232, 2012.

[23] T. Dong, X. Liao, and T. Huang, "Bogdanov-Takens bifurcation in a tri-neuron BAM neural network model with multiple delays," Nonlinear Dynamics, vol. 71, no. 3, pp. 583-559, 2013. 


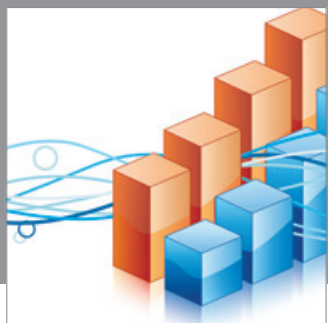

Advances in

Operations Research

mansans

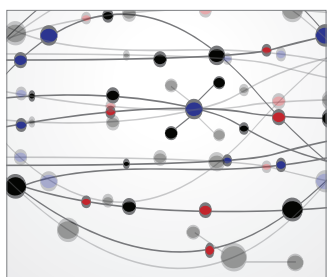

The Scientific World Journal
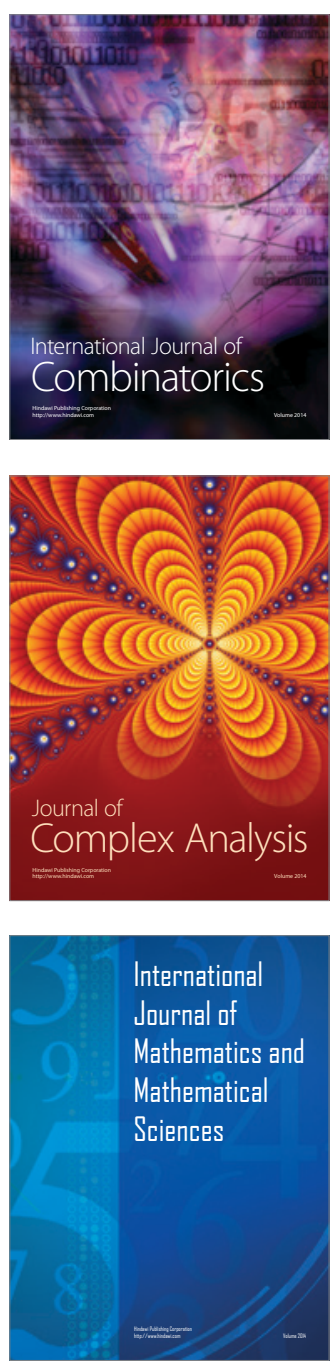
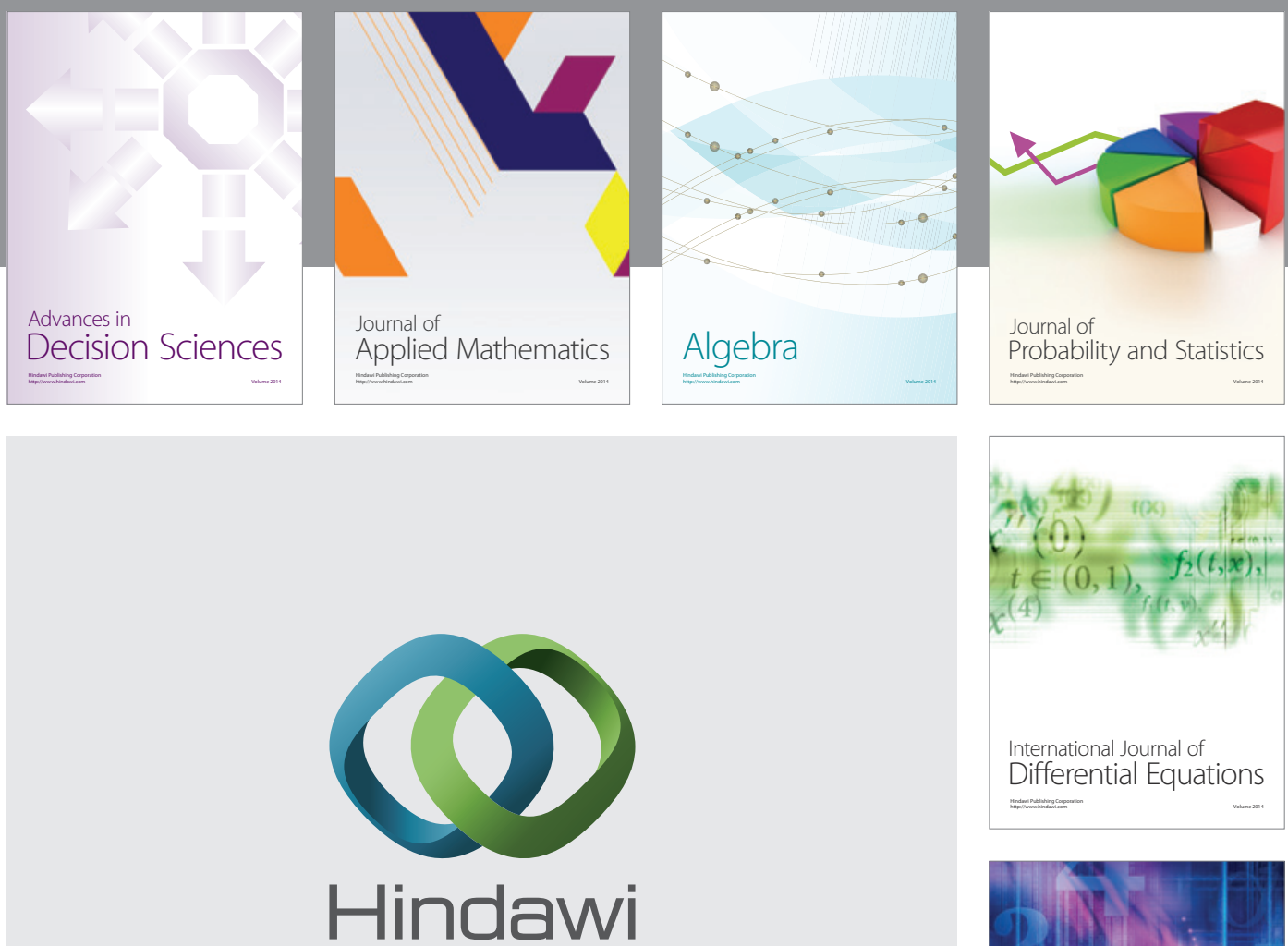

Submit your manuscripts at http://www.hindawi.com
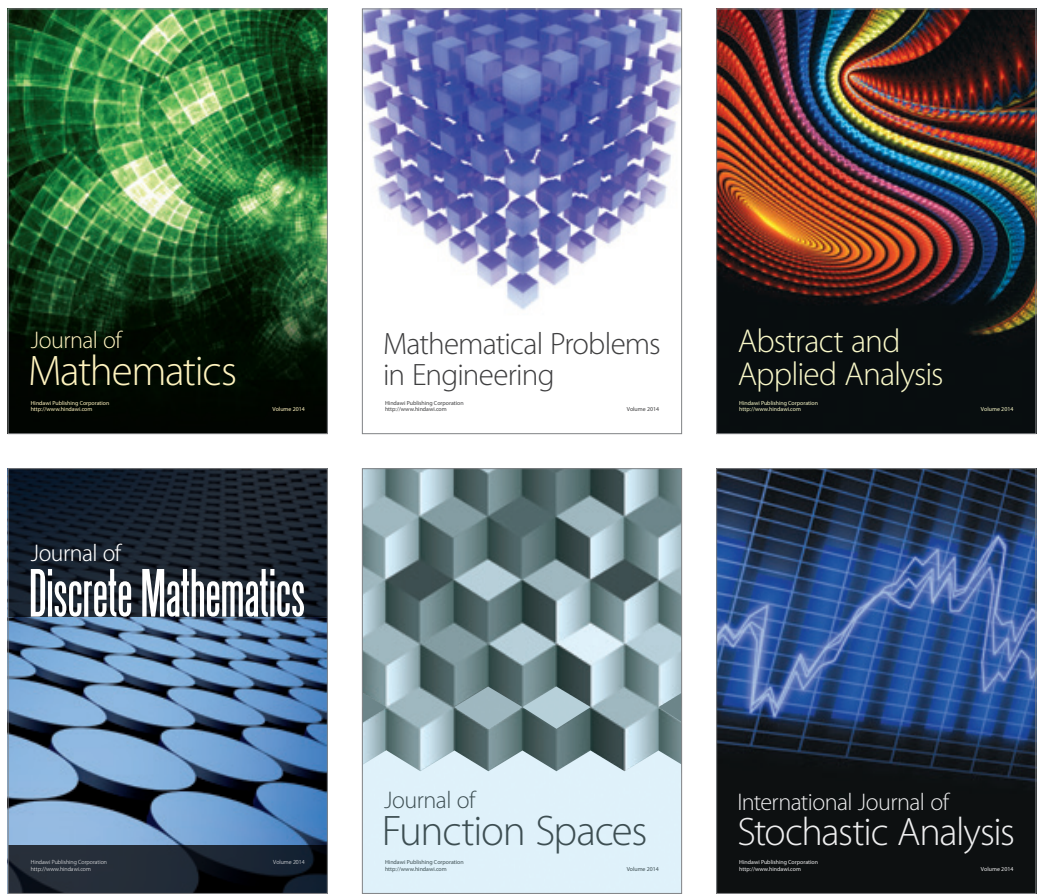

Journal of

Function Spaces

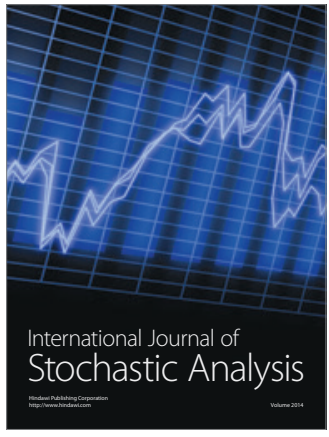

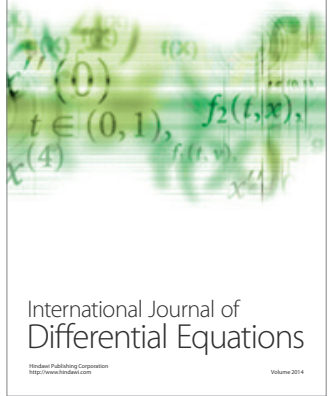
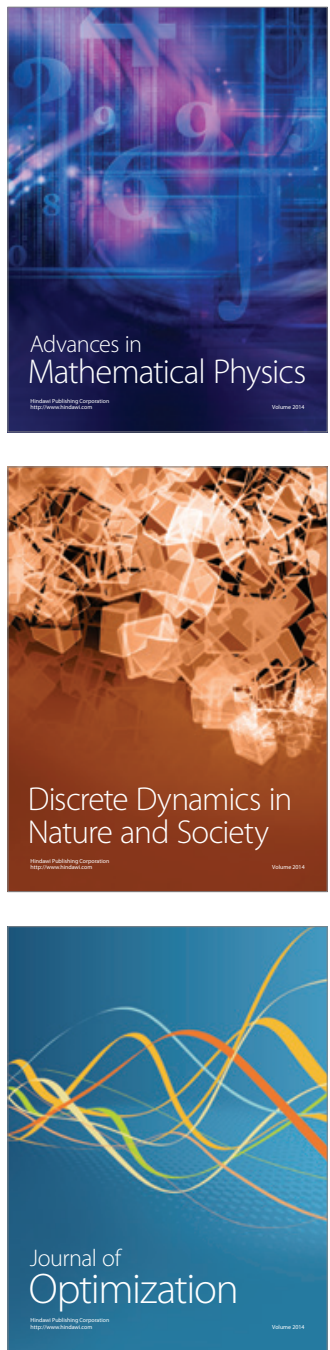